\title{
9 Die Krim zwischen der Kiewer Rus', Byzanz und seminomadischen Gruppen aus dem eurasischen Raum
}

\begin{abstract}
Ein [...] Teil des Volkes der Petschenegen lebt neben dem Gebiet von Cherson; diese treiben mit den Chersoniten Handel und verrichten Dienst für sie [die Chersoniten] und den Kaiser, und zwar in Rußland, Chazaria und Zichia und in allen Gebieten jenseits davon, das heißt sie erhalten von den Chersoniten den vorher für diesen Dienst vereinbarten Lohn, wie er der Arbeit und Mühe entspricht, in der Form von Purpurkleidern, Seidentüchern, Seidenstoffen, Gürteln, Pfeffer, echtem ,Parthischem ' Leder und anderen Waren, die von ihnen verlangt werden, je nach dem zu welcher Vereinbarung ein Chersonit einen Petschenegen überredet oder von diesem überredet wird. Denn weil die Petschenegen frei und sozusagen ihre eigenen Herren sind, verrichten sie nie einen Dienst ohne Lohn. ${ }^{1}$
\end{abstract}

In diesen ebenfalls aus der Lehrschrift „De administrando imperio“ stammenden Zeilen werden einige Güter genannt (Gewürze, Stoffe etc.), die in der Ökonomie des Schwarzmeerraumes eine Rolle spielten und für die Chersones ein Umschlagplatz war. Überdies geben sie Auskunft über die ökonomischen Verflechtungen und Abhängigkeiten zwischen dem byzantinischen Außenposten Chersones, den Petschenegen und der Rus', die in der obigen Übersetzung aus dem Griechischen anachronistisch „Rußland“ genannt wird. Die Petschenegen, die im 9. und 10. Jahrhundert weite Teile der heute russischen und ukrainischen südlichen Steppengebiete einschließlich der nördlichen Krim kontrollierten, waren ein weiterer aus dem asiatischen Raum vorgedrungener polyethnischer, multilingualer (überwiegend turksprachiger) Personenverband bzw. eine „nonterritorial professional community“, so der Mediävist Omeljan Pritsak. ${ }^{2}$ Für die Wirtschaft und das politische Gleichgewicht im Schwarzmeerraum waren sie von großer Relevanz; nicht umsonst heißt es in Konstantins Regierungsanweisung an seinen Nachfolger, dass „es für den Kaiser der Romäer stets von großem Nutzen ist, mit dem Volk der Petschenegen (Patzinakitai) Frieden halten zu wollen, freundschaftliche Vereinbarungen und Verträge zu schließen, jedes Jahr zu ihnen einen Gesandten mit angemessenen und passenden Geschenken zu schicken.“3 Auch die BewohnerInnen der Rus' seien am Einvernehmen mit ihnen interessiert, denn „sie kaufen Rinder, Pferde und Schafe und sie leben davon leichter und

1 Belke u. Soustal (1995), 75.

2 Pritsak (1975), 228.

3 Belke u. Soustal (1995), 71.

๑ OpenAccess. ( 2020 Kerstin S. Jobst, publiziert von De Gruyter. (cc))BY Dieses Werk ist lizenziert unter der Creative Commons Attribution 4.0 International. https://doi.org/10.1515/9783110520620-011 
angenehmer, weil es keines der genannten Tiere in Rußland gibt. “4 Die Petschenegen ihrerseits strebten ebenfalls nach pragmatischen Übereinkünften mit den Nachbarn und betrieben - wie in der Region ja auch in früheren Zeiten üblich eine flexible Bündnispolitik, schlossen Pakte mit Byzanz (z. B. 914, 968, 972), der Rus' (944) und anderen Anrainern. ${ }^{5} \mathrm{Zu}$ dieser Flexibilität gehörten aber auch sporadische Einfälle gegen diese, so etwa im Jahr 968, von dem es in der „Nestorchronik“ heißt: „Und die Pečenegen umgaben die Stadt [Kiew] mit großer Streitmacht, eine zahllose Menge rings um die Stadt; und es war nicht möglich, aus der Stadt hinauszugehen noch Nachricht $\mathrm{zu}$ senden. Die Leute wurden schwach vor Hunger und [Mangel an] Wasser."6 Im Jahr 972 erlitt die Rus' schließlich eine vernichtende Niederlage gegen die Petschenegen: „Svjatosláv [zog] zu den Schwellen, und Kurja, ein Fürst der Pečenegen, überfiel ihn. Und sie erschlugen den Svjatosláv, und sie nahmen seinen Kopf, und aus seinem Schädel machten sie einen Pokal, indem sie den Schädel umschmiedeten, und sie tranken aus ihm." ${ }^{\text {"7 }}$

Diese und weitere Auseinandersetzungen fanden zwar nördlich der Halbinsel in den Steppengebieten oder auch im Zentrum der Rus' - so die Belagerung Kiews 968 oder die Schlacht um Kiew 1036 - statt, hatten aber dennoch Auswirkungen auf die Krim. Zwischen dem ausgehenden 9. bis ins 11. Jahrhundert hinein fielen Petschenegen (zum Teil in Verbund mit anderen aus dem eurasischen Raum stammenden Verbänden) immer wieder auf der Halbinsel ein. Zu Beginn des 11. Jahrhunderts zeigte sich wieder einmal, dass vor allen Dingen die nördliche Krim und die angrenzenden Gebiete auf dem Festland ein Durchzugsgebiet waren, so dass sich dort keine zentrale Macht etablieren konnte. Hingegen stand der Süden bzw. der Südwesten (Chersones einschließlich des Umlandes sowie KrimGotthia) weiterhin unter dem Einfluss Byzanz'. Auch die Petschenegen-Herrschaft sollte nur eine vorübergehende sein. 1036 gelang dem Großfürsten der Rus', Jaroslav I. („Mudryj“ [der Weise], um 978-1054), nämlich ein bedeutender Sieg gegen diese, was in der Rückschau als Zeichen gedeutet werden muss, dass deren Zenit überschritten war. Im Süden versuchten sie zwar noch vereinzelte Angriffe Richtung Konstantinopel, erlitten aber empfindliche Niederlagen. Und im Osten bedrohten neue mobile Gruppen diesen Verband, welcher schließlich nach Westen in das Donaugebiet abzog bzw. sich an andere BewohnerInnen akkultu-

4 Belke u. Soustal (1995), 72.

5 Pritsak (1975), 232.

6 Müller L. (2001), 80.

7 Müller L. (2001), 90. Das Motiv, aus den abgeschlagenen Köpfen der Gegner Trinkbecher zu machen, ist uns bereits in Herodots Skythenbeschreibung begegnet (vgl. Kapitel 3). 
rierte. Eine letzte Erwähnung fanden die Petschenegen in russischen Chroniken unter dem Jahr $1169 .^{8}$

Das Vordringen der Rus' in Richtung Schwarzes Meer war kein linearer Prozess, sondern erfolgte phasenweise. Soweit quellenmäßig erfassbar, kam es seit der Mitte des 9. Jahrhunderts sporadisch zu (teils friedlichen, teils militärischen) Kontakten mit dem Chaganat und Byzanz, die später „systematisch“ wurden. ${ }^{9}$ Ein Ereignis von großer Wirkung bis in unsere Zeit - zumindest auf der diskursiven Ebene - war die eingangs geschilderte (vgl. Kapitel 2) Episode um das Jahr 988 herum, als der Großfürst von Kiew aus dem Geschlecht der Rjurikiden, Vladimir/ Volodymyr, unter nicht ganz geklärten Umständen Chersones mit seinen Truppen erst belagerte, dann (vermutlich) dort die christliche Taufe erhielt und schließlich die Ehe mit Anna Porphyrogenneta, der Schwester der Kaiser von Konstantinopel, einging; in jedem Fall bedeuteten die Taufe und die Heirat mit einer purpurgeborenen Prinzessin einen erheblichen Prestigegewinn für die bis dahin heidnische Rus'. Dass sich Byzanz auf diese Verbindung überhaupt eingelassen hatte, ist ein Indiz für die Bedeutung, die der Rus' beigemessen wurde. Diese hatte im Gegensatz zu den meisten bisherigen Akteuren, die sich auf dem Land fortbewegten, nämlich „dauerhaften Zugang zu Schiffsressourcen und [setzten] diese auch ein. “10 Friedliche Handelskontakte zwischen der Rus’ und Byzanz, die sich aber weiterhin sporadisch mit Raubzügen Kiews gegen Konstantinopel abwechselten, prägten die Beziehungen vor allem seit dem 10. Jahrhundert. In diesem Zusammenhang ist noch auf die Existenz eines aus der griechischen Gründung Hermonassa hervorgegangenen slavischen Fürstentums auf der Pantikapaion/ Kerč' gegenüberliegenden Halbinsel Taman hinzuweisen. Dieses trug den Namen Tmutarakan' und war Ende des 10., Anfang des 11. Jahrhunderts unter die Herrschaft eines Zweigs der Rjurikiden-Dynastie gelangt. Der „Nestorchronik“ zufolge nahm einer der Söhne Vladimirs, Mstislav (um 988-1034/36), die Stadt nach einem siegreichen Ringkampf mit dem bis dahin Tmutarakan' beherrschenden Fürsten Rededja ein. Dieser hatte vorgeschlagen, nicht die Heere, sondern die beiden Heerführer gegeneinander antreten zu lassen, „[u]nd wenn du gewinnst, so magst du meine Habe und mein Weib und meine Kinder und mein Land nehmen; wenn aber ich gewinne, so nehme ich all das Deine“, so das Angebot Rededjas. Als Mstislav den Zweikampf zu verlieren drohte, rief dieser die Mutter

8 Pritsak (1975), 231.

9 Pletnjowa (1978), 133-135.

10 Albrecht u. Herdick (2013), 34. Vgl. auch die lebendige Beschreibung des Vordringens der Krieger der Rus’ auf dem Wasserweg unter der Überschrift „Über die Russen, die mit Einbäumen aus Rußland nach Konstantinopel kommen“ in „De administrando imperio“. Belke u. Soustal (1995), 78-86. 


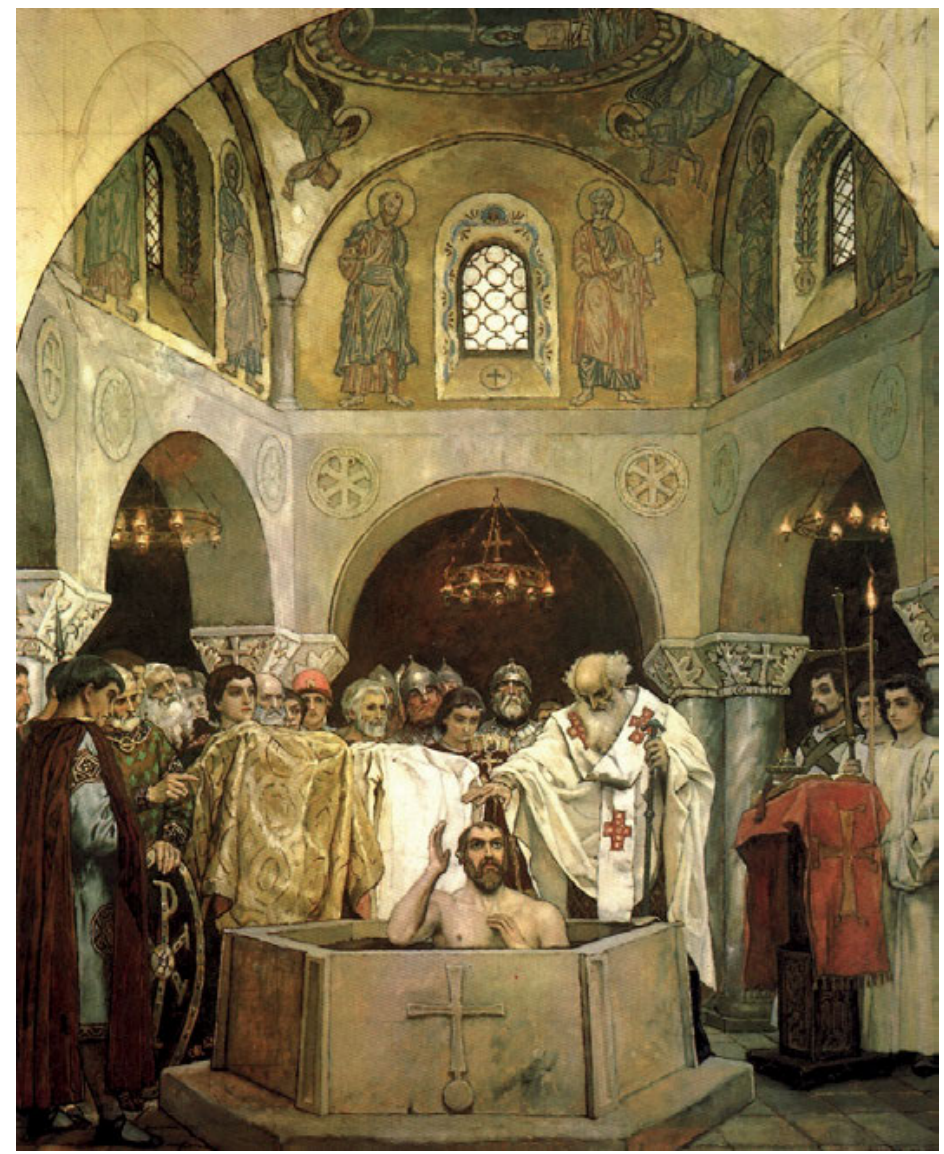

Abb. 5: Taufe Vladimirs, Gemälde von Viktor Vasnecov, 1890

Gottes um Beistand an - und er „schlachtete den Rededja.“11 Diese der Quelle zufolge mit göttlichem Beistand erlangte Herrschaft über Tmutarakan' währte allerdings nicht lange, denn schon im 12. Jahrhundert wurde durch das Vordringen der Kumanen (siehe Kapitel 10) der Niedergang des Fürstentums eingeleitet. 1094 wird es letztmalig in russischen Chroniken erwähnt. In der Folge geriet es unter unterschiedliche Herrschaften. ${ }^{12}$ Die Konstellation - sporadisches und streckenweise erfolgreiches Vordringen der Rus' nach Süden, die Existenz Tmu-

11 Müller L. (2001), 181.

12 Dazu im Überblick Čchaidze (2010). 
tarakan's an der Meerenge von Kerč' - wurde in der russischsprachigen Geschichtswissenschaft, nicht nur des 19. Jahrhunderts, immer wieder dahingehend interpretiert, dass der nördliche Schwarzmeerraum und vor allen Dingen auch die Krim schon seit dem 9./10. Jahrhundert einem dauerhaften slavischen Einfluss ausgesetzt gewesen sei. Diese Lesart passte nicht zuletzt seit dem ausgehenden 18. Jahrhundert den Apologeten russischer Herrschaft über die Region ins Kalkül, da man glaubte, so die Annexion der Krim von 1783 quasi als die Wiedergewinnung alten slavischen Bodens interpretieren zu können. ${ }^{13}$ Nicht von einem relevanten Anteil slavischer BewohnerInnen auf der Halbinsel, aber immerhin von einem kurzlebigen Protektorat der Rus' über Krim-Gotthia in den Jahren 962 bis 972 geht mit Vasil'ev selbst eine unbestrittene Autorität der mittelalterlichen Geschichte der Krim aus. ${ }^{14}$ Eine andere Autorität, der bedeutende russische Historiker Vasilij O. Ključevskij (1841-1911), widersprach hingegen derartigen Auffassungen. ${ }^{15}$

13 So z. B. dem russischem Universalgelehrten Michail V. Lomonosov (1711-1765), der Mitte des 18. Jahrhunderts sogar glaubte beweisen zu können, dass die Sarmaten eigentlich Slaven gewesen seien; demnach hätten ,Slaven` bereits in den Jahrhunderten vor dem Beginn unserer Zeitrechnung in der Region gelebt. Vgl. Slezkine (1997), 50 und 57. Zu den Versuchen Stalins, für die Region den Beweis einer autochthonen slavischen Bevölkerung zu führen, vgl. Ascherson (1996), $74 \mathrm{f}$. 14 Vasiliev (1936), 118-126.

15 Kliutschewskij (1925ff.), Bd. 1., 99. 
